\title{
L'Institut national du cancer : un institut dédié au cancer pour garantir une impulsion forte et continue de la lutte contre cette maladie
}

\author{
The French National Cancer Institute (INCa): an institute dedicated to a strong and lasting \\ action in the fight against this disease
}

\author{
A. Burstin \\ (C) Springer-Verlag France et INCa 2014
}

Résumé L'Institut national du cancer (INCa) est l'agence sanitaire et scientifique chargée de coordonner les actions de lutte contre le cancer. Créé par la loi de santé publique de 2004, l'Institut est un modèle original et unique tant parmi les opérateurs de l'État qu'à l'échelle internationale, avec un périmètre couvrant l'ensemble des modes d'action face à la maladie : la recherche, l'observation et l'évaluation, la prévention et le dépistage, l'organisation des soins et l'information des publics. Coordination et intégration de l'ensemble des leviers d'intervention sont les fondements de l'action de l'Institut. La coordination des actions de lutte contre le cancer est une mission majeure de l'INCa, traduite dans son statut de groupement d'intérêt public (GIP) qui fédère les principaux acteurs du champ et par la mobilisation de l'ensemble des intervenants, publics, associatifs, professionnels ou industriels. Cet échange constant avec les acteurs de la cancérologie et l'intégration des différents axes de la lutte contre le cancer sont garants de l'expertise et de la pertinence des actions que l'Institut impulse. La responsabilisation inhérente à une institution dédiée aux pathologies les plus meurtrières dans notre pays est un autre élément clé, qui conduit l'INCa à assumer le pilotage du Plan cancer 2014-2019, en lien étroit avec les ministères chargé de la Santé et de la Recherche.

Mots clés Institut national du cancer - Lutte contre le cancer . Agence sanitaire - Plan cancer - Recherche - Observation . Prévention · Dépistage · Organisation des soins · Information.

Abstract The French National Cancer Institute (INCa) is the preeminent health and science agency in charge of

\footnotetext{
A. Burstin $(\square)$

Directrice générale de l'Institut national du cancer Institut national du cancer, 52 avenue André Morizet F-92513 Boulogne-Billancourt Cedex
}

cancer control. Created under the Public Health Act of 2004, the French National Cancer Institute is an original and unique model among State agencies and at international level; it covers all modes of action to fight against the disease: research, observation and evaluation, prevention and screening, care organization and public information. The Institutes' work is based on coordinating and integrating all intervention programmes. This coordination is a major mission of INCa and translates in its status of public interest group: it gathers all key stakeholders -charities, public sector, professional, industries- and favours constant exchanges. Along with the integration of all aspects of the fight against cancer, they warrants the expertise and relevance of INCa-driven actions. The inherent liability of an institute dedicated to the most deadly diseases in France is another key element that leads INCa to steer the 2014-2019 Cancer Plan, in close collaboration with the Ministries for Health and Research.

Keywords French National Cancer Institute $\cdot$ Fight against cancer · Health agency · French Cancer Plan - Research . Observation - Prevention - Screening - Care organization · Public information.

Suite aux travaux de la commission nationale d'orientation sur le cancer en 2003, la lutte contre le cancer est déclarée « impératif national » ${ }^{1}$. La nécessité d'une structure globale d'impulsion et de pilotage stratégique conduit à la création d'un Institut national du cancer (INCa). Dédiée à une pathologie, cette agence sanitaire et scientifique chargée de coordonner les actions de lutte contre le cancer instaure un modèle original et unique parmi les opérateurs de l'État avec un périmètre d'action couvrant l'ensemble des leviers d'intervention face aux cancers : la recherche, l'observation

\footnotetext{
${ }^{1}$ Rapport sur le projet de loi relatif à la politique de santé publique, JM Dubernard, 25 septembre 2003.
} 
et l'évaluation, la prévention et le dépistage, l'organisation des soins, l'information des publics. ${ }^{2}$ Ce modèle est également une originalité à l'échelle internationale, y compris par rapport aux instituts américains, dont le National Cancer Institute, qui n'ont à intervenir que dans les champs de la recherche et des recommandations.

Conçu comme un ensemble cohérent de leviers d'action complémentaires imposés par la complexité de cette pathologie, l'INCa est porteur d'importantes ambitions ; celles-ci sont dessinées notamment dans le rapport de la commission nationale d'orientation sur le cancer :

- définition et coordination d'une politique nationale de recherche novatrice en cancérologie couvrant tous les champs, dont les sciences humaines ;

- référence scientifique dans tous les domaines de la prévention au dépistage et à la prise en charge du cancer ;

- veille sur la performance du système de santé dans le domaine du cancer ;

- délivrance d'informations validées pour tous les publics ;

- impulsion pour une initiative européenne forte contre le cancer.

Le rapport parlementaire relatif à la loi de santé publique qui crée l'institut résume ces enjeux par trois mots clés : impulsion, coordination, qualité. Auxquels on doit ajouter, avec près de dix ans de recul désormais, une quatrième notion, celle d'intégration des différentes facettes de la lutte contre le cancer.

Ces quatre termes résument bien ce qui légitime et motive la création d'une institution dédiée à une pathologie et ce au regard de quoi elle devra à terme être jugée : la responsabilisation inhérente à une institution dédiée garantit l'investissement dans la durée nécessaire à une impulsion forte et continue ; la coordination des acteurs est une mission majeure de l'INCa, traduite symboliquement dans son statut de groupement d'intérêt public (GIP) qui a vocation à fédérer les principaux acteurs publics et privés du champ ${ }^{3}$ pour surmonter la dispersion des efforts de lutte contre le cancer ; elle s'exprime aussi plus largement dans la mobilisation d'acteurs publics, associatifs, professionnels ou

\footnotetext{
${ }^{2}$ La loi du 11 août 2004 confie à l'INCa un champ de compétence couvrant tous les domaines d'intervention, faisant en cela suite à l'une des préconisations du rapport de la commission nationale d'orientation sur le cancer de janvier 2003 recommandant la création d'un institut national doté de 5 principales missions : recherche, expertise, observation et évaluation, information du public et action européenne et internationale. L'INCa est placé sous la tutelle conjointe des ministères chargés de la santé et de la recherche.

${ }^{3}$ La loi a créé l'INCa sous forme d'un groupement d'intérêt public (GIP) qui réunit les ministères chargés de la santé et de la recherche, les associations (Ligue nationale contre le cancer, Fondation ARC pour la recherche sur le cancer), les caisses nationales d'assurance-maladie (CNAMTS,MSA, RSI), les principaux organismes de recherche (INSERM, CNRS) et les fédérations hospitalières (FHF, FHP, FEHAP, FNCLCC).
}

d'industriels. L'intégration en son sein des différents axes de la lutte contre le cancer est un élément clé de l'expertise et de la pertinence des actions : une telle structure peut et doit enrichir l'action en santé publique des enseignements de la recherche fondamentale ou interventionnelle comme de l'observation des faits. Impulsion, coordination et intégration sont trois leviers au service de l'amélioration de la qualité des prises en charge préventives ou curatives, indispensable à la réduction de la mortalité et de la morbidité imputables au cancer.

\section{Impulsion et coordination}

La nécessité de coordonner les efforts de multiples acteurs pour conjuguer leurs leviers d'action et leurs moyens, tout particulièrement en matière de recherche, est à la source de la création de l'INCa. Face à la complexité d'une maladie qui continue à défier la recherche et la médecine, la dispersion des efforts comme l'absence de stratégie unifiée constituent des handicaps inacceptables. C'est cette même logique qui conduit aujourd'hui les différents pays à unir leurs connaissances au sein d'une Global Alliance pour le partage des données génomiques et cliniques en cours de construction. Mais cette union des forces a également tout son sens en matière de prévention - lutte contre les comportements à risque notamment - ou de soins, concertation indispensable des professionnels et des institutions en faveur de la qualité.

Pour manifester l'union des acteurs majeurs de la lutte contre le cancer, l'INCa est constitué sous forme d'un groupement d'intérêt public (GIP), unissant notamment les ministères de la santé et de la recherche, les grandes associations - Ligue contre le cancer et Fondation ARC, l'ensemble des fédérations hospitalières et les principaux régimes d'assurance maladie, les grands organismes de recherche, INSERM et CNRS. Pour consacrer la nécessité d'une stratégie coordonnée de recherche, un principe d'unité de direction entre le pôle Recherche de l'INCa et l'ITMO Cancer de l'Alliance pour les sciences de la vie et la santé (Aviesan) est posé, qui conduit à l'élaboration d'une stratégie pluriannuelle commune. Pour décliner cette stratégie validée par le conseil scientifique et le conseil d'administration de l'INCa, les moyens des deux grandes associations ARC et Ligue sont conjugués à ceux de l'Institut dans le cadre de grands programmes thématiques de recherche intégrée et les moyens de la recherche clinique et de la recherche translationnelle du ministère de la Santé sont mobilisés par l'Institut pour leur part dédiée au cancer.

La conjonction des efforts des membres du GIP bénéficient à d'autres pans de la lutte contre le cancer : l'information des patients, qui unit les efforts notamment de la Ligue et de l'INCa, le soutien à l'innovation, qui conjugue 
les forces de l'Institut, des ministères de la santé et de la recherche, des grands organismes de recherche et des fédérations hospitalières ; la communication sur le dépistage soutenue par le ministère de la santé et les régimes d'assurance maladie...

Le rôle de coordination de l'INCa ne se limite toutefois pas aux membres du GIP : d'une part, l'INCa a un rôle de pilotage technique de structures oeuvrant dans le champ du cancer, par exemple les réseaux régionaux de cancérologie ou les cancéropôles, pour lesquels le $3^{\text {e }}$ Plan cancer renforce son rôle d'animation dans une logique de décloisonnement des acteurs.

D'autre part, en travaillant depuis longtemps à la concertation avec de nombreuses associations de malades, notamment autour de la plateforme Cancer info, en mobilisant associations et sociétés savantes dans ses travaux relatifs aux bonnes pratiques ou à la recherche, en encourageant par des appels à projets dédiés la fédération de groupes coopérateurs de recherche pour permettre le déploiement d'essais cliniques plus larges et plus ambitieux... l'INCa rassemble les acteurs du soin et de la recherche et les usagers pour optimiser leurs interventions. Plus récemment, il a cherché lors de la préparation du $3^{\text {e }}$ Plan cancer à mobiliser les partenaires sociaux, acteurs majeurs de la réinsertion professionnelle de malades trop souvent pénalisés dans le monde du travail.

Ce rôle de coordination et de rapprochement des acteurs s'illustre également dans les interactions de l'INCa avec les industriels de santé : au sein des centres d'essais de phase précoce comme du programme AcSé (pour « accès sécurisé aux molécules innovantes »), l'INCa amène industriels et promoteurs académiques à coopérer pour accélérer les progrès de la recherche et l'accès des patients aux molécules innovantes. En envisageant désormais des essais associant plusieurs molécules issues de différents laboratoires, l'INCa montre que la poursuite tenace d'une stratégie partagée est source de décloisonnement et de coopération.

Porteur d'une approche par pathologie, l'Institut doit, au-delà de la coordination des acteurs de la lutte contre le cancer, constamment veiller à son articulation avec les acteurs généralistes de la santé, nationaux ou régionaux comme les ARS. Cela vaut notamment pour les agences sanitaires dans le champ de la prévention (INPES), de la surveillance (InVS), de la qualité des pratiques et du bon usage du médicament (HAS). Le succès de la dialectique constante entre spécificité Cancer et approche généraliste suppose à la fois clarification des rôles, qui est recherchée au travers d'accords-cadre et volonté de coopération traduite par des plans d'actions communs. Destiné à la coordination, l'INCa est aussi voué, par construction, à interagir avec les autres opérateurs publics dans chacun des champs d'action qu'il partage avec eux. Il a pu parfois être pour cela remis en question. Sa légitimité et sa force résident pourtant dans la conjonction de toutes ces actions au sein d'une même institution et dans sa démarche intégrée.

\section{Intégration}

La valeur ajoutée d'une institution dédiée au cancer et son rôle de référence scientifique tiennent en effet très largement à sa capacité à croiser les enseignements de la recherche, de l'observation et de l'évaluation et à traduire immédiatement cette connaissance en expertise sous forme de recommandations pour les pouvoirs publics ou les professionnels, de propositions d'organisations des soins/de la prévention et d'information du public. Point de rassemblement des connaissances sur le cancer, l'Institut doit s'en servir pour orienter son action d'agence de moyens pour la recherche, pour proposer la structuration la plus pertinente de la prise en charge des patients ou de la recherche de pointe, pour rendre accessible au grand public une information de référence.

Cette vision transversale est un atout exceptionnel propre à lui permettre d'éclairer des institutions généralistes « verticales » qui ne maîtrisent par définition qu'une partie des enjeux. Grâce à sa capacité à décloisonner les sujets, à susciter des interactions précoces et fécondes, particulièrement entre recherche et soins, entre recherche en santé publique et prévention/dépistage, mais aussi entre prévention et soins, une institution dédiée est source d'une incontestable valeur ajoutée. La mise en responsabilité d'une institution incarnant symboliquement la volonté d'action collective a également des effets induits bénéfiques, en créant une obligation de vigilance et de réactivité qui conduit l'Institut à prendre des initiatives dont la nécessité lui apparait plus clairement qu'à d'autres institutions ayant une vision plus partielle des sujets.

Plusieurs exemples peuvent illustrer ces interactions fécondes et structurantes qui fondent désormais de plus en plus systématiquement le plan d'action annuel de l'Institut.

En 2013, l'ensemble des directions de l'INCa ont ainsi été mobilisées sur le cancer du sein, compte tenu des enjeux de santé publique, en lien avec les débats autour du dépistage organisé et des risques de sur-traitement. Cette démarche transversale a associé une actualisation des connaissances sur les bénéfices et les limites du dépistage, la mise en place d'outils permettant de progresser dans l'évaluation des pratiques afin d'apprécier un éventuel sur-traitement (soutien à l'observatoire national des carcinomes canalaires in situ, analyse à partir des données médico-économiques des trajectoires des femmes), la diffusion de recommandations de bonne pratique actualisées sur la prise en charge des cancers du sein in situ, une campagne d'information sur le dépistage revue pour mieux éclairer les femmes, et enfin, un programme intégré de recherche sur le cancer du sein. L'animation transversale de l'ensemble des collaborateurs 
de l'Institut impliqués dans cette thématique a permis de préciser la stratégie d'action, de repérer des manques, d'accroître les collaborations avec des partenaires communs.

Le plan d'action 2015 envisagera dans le même esprit décloisonné une action transversale forte pour lutter contre les cancers de l'enfant, en renforçant les liens avec les professionnels et les associations concernés, en confortant les réseaux de soins spécialisés et en les mobilisant plus activement au profit de la recherche, en étendant la démarche d'essais de phase précoce à la pédiatrie, en diffusant enfin des documents pédagogiques sur les cancers de l'enfant éclairant les parents sur la prise en charge mais aussi sur la recherche clinique.

Un autre domaine illustre de façon exemplaire la fructification croisée des différents leviers d'action de l'INCa et son potentiel cumulatif : il s'agit de la génétique moléculaire. Témoin, au travers de ses appels à projets de recherche, des potentialités croissantes de la génétique moléculaire, l'Institut a œuvré à la structuration sur le territoire national de 28 plateformes d'analyse moléculaire : les tests qui y sont réalisés permettent d'identifier une anomalie moléculaire spécifique d'une tumeur et ainsi, de délivrer une thérapie ciblée aux patients qui le nécessitent. Les mêmes plateformes mettent à disposition des tests issus de l'identification par la recherche de marqueurs de prédisposition génétique au cancer, permettant une prise en charge préventive au sein des consultations d'oncogénétique organisées par l'INCa. Celui-ci produit en outre des recommandations de prévention ciblée en lien avec ces prédispositions. Au moment où s'affirme par ailleurs le concept de chimio-prévention basée sur les connaissances permises par la génétique moléculaire, ces acquis seront précieux pour développer cette nouvelle facette de l'action préventive.

Les plateformes ont permis à la France de disposer d'un accès précoce et envié à cette technique, contribuant aux progrès français majeurs de la médecine personnalisée. Cette structuration de prise en charge a permis d'amplifier la recherche et de doper les travaux des équipes françaises qui ont manifesté au dernier grand congrès de l'ASCO de leur dynamisme dans ce champ majeur de l'innovation en cancérologie. Le diagnostic moléculaire des tumeurs par les plateformes a en effet permis de favoriser de façon tout à fait remarquable l'inclusion des patients français dans des essais cliniques de phases précoces réalisés par les CLIP $^{2}$ qui évaluent le plus souvent des thérapeutiques innovantes de type thérapies ciblées (plus 100\% de patients français participant à des essais de phase I/II en 2 ans). Les essais cliniques de phase précoce réalisés dans les CLIP ${ }^{2}$ sont souvent dédiés aux cancers rares, les files actives de malades étant celles des réseaux de prise en charge des cancers rares labellisés par l'INCa. La connaissance épidémiologique permise par ces réseaux nourrit donc également la recherche, facilitée par les bases clinicobiologiques financées par l'INCa qui leur sont associées.

Autre exemple de la valeur ajoutée d'une institution veillant constamment à valoriser sa connaissance du cancer et de sa prise en charge pour éclairer les politiques, la constitution très prometteuse d'une Cohorte Cancer réunissant l'ensemble des données anonymisées relatives aux personnes atteintes de cancer : permettant d'analyser les trajectoires de prise en charge et les pratiques, cette cohorte va apporter d'indispensables informations pour orienter la production de recommandations de bonne pratique, pour définir des indicateurs clés de qualité, pour imaginer de nouveaux modes de financement innovants (au parcours par exemple). Elle sera totalement emblématique de l'enrichissement constant de l'action par la connaissance permise par le rassemblement de ces deux dimensions au sein d'une institution unique.

Point fort de l'INCa, l'intégration des différentes approches du cancer permet aussi de répondre au mieux aux saisines ministérielles qui mobilisent généralement plusieurs directions (données d'observation, connaissance des organisations, de la démographie et des pratiques professionnelles, état de l'art...).

Coordination des acteurs et intégration des différentes facettes de la lutte contre le cancer sont les lignes de force de l'action de l'INCa. Elles lui confèrent une capacité à anticiper les enjeux à venir mais aussi une responsabilité majeure : parce qu'il se nourrit du dialogue avec l'ensemble des acteurs de la cancérologie et parce qu'il peut influer sur bien des dimensions de la lutte contre cette pathologie, l'INCa ne peut éluder les questions difficiles. Il est investi d'une mission exigeante qui est de porter haut l'ambition de la lutte contre le cancer et d'être un constant aiguillon des autres acteurs, qu'ils appartiennent au champ sanitaire ou au domaine plus large des parties prenantes de l'après-cancer (partenaires sociaux, école, acteurs sociaux ou monde de l'assurance). Cette mission l'a conduit à assumer le pilotage du $3^{\text {e }}$ Plan cancer et à veiller à ce que ses ambitions en matière de recherche, de soins et d'amélioration des conditions de l'après-cancer soient traduites en actions. 